elements, and also of such radiation as originates from the outer levels of compound and other aggregate formations.

Physics Department,

University of Allahabad, July 10.

IN continuation of my previous notes in NATURE (May 17 and June 7, 1930), I have further observed the following lines:

\begin{tabular}{|c|c|c|c|}
\hline $\begin{array}{c}\text { Incident } \\
\text { Radiation. }\end{array}$ & $\begin{array}{l}\text { Scattering } \\
\text { Substance. }\end{array}$ & Modified Lines. & Origin. \\
\hline $\begin{array}{c}\mathrm{NiK} \beta_{1} \\
\nu / R=608 \cdot 7\end{array}$ & $\begin{array}{l}\mathrm{C} \\
\mathrm{N} \\
\mathrm{O}\end{array}$ & $\left.\begin{array}{r}1552 \mathrm{X} . \mathrm{U} . \\
(587 \cdot 0) \\
1573 \mathrm{X} . \mathrm{U} . \\
(579 \cdot 0) \\
1602 \mathrm{X} . \mathrm{U} . \\
(569 \cdot 0)\end{array}\right\}$ & $\begin{array}{l}\mathrm{Ni} K \beta_{1}-\mathrm{C} K a \\
\mathrm{Ni} K \beta_{1}-\mathrm{N} K a \\
\mathrm{Ni} K \beta_{1}-\mathrm{O} K a\end{array}$ \\
\hline $\begin{array}{c}\mathrm{Fe} K \beta_{1} \\
\nu / R=519 \cdot 9\end{array}$ & $\mathrm{~N}$ & $\left.\begin{array}{r}1825 \mathrm{X} . \mathrm{U} . \\
(499 \cdot 0) \\
1859 \mathrm{X} . \mathrm{U} . \\
(490 \cdot 0)\end{array}\right\}$ & $\begin{array}{l}\mathrm{Fe} K \beta_{1}-\mathrm{C} K a \\
\mathrm{Fe} K \beta_{1}-\mathrm{N} K a\end{array}$ \\
\hline
\end{tabular}

The values of the $K a$ radiations (in $\nu / R$ ) of carbon, nitrogen, and oxygen are $20 \cdot 4,28 \cdot 7$, and $38 \cdot 3$ respectively (Söderman, Zeit. f. Phys., 52). These modified lines are broad, diffuse, and weak, and as such they were only measured by a glass scale. The error in the measurement may be so great as 4 X.U.

Though Coster, Ehrenberg, and Kast (as mentioned in my previous notes), following Bergen Davis and his collaborators, who were the first to report the detection of such modified lines in the scattered rays, have failed to detect any line on the photographic plate, through the scattering of $\mathrm{X}$-rays by an atom in a direction at right angles to the direction of propagation, where the Raman effect is usually observed, this experiment clearly shows that the modified lines produced by the "scattering of X-rays by bound electrons " are observed in the direction of transmission of the incident radiation. It further follows that to be consistent with the current definition of scattering and absorption, the effect observed by me, which was described in my previous notes to NATURE as "modified lines due to the scattering of X-rays by bound electrons", would be more correctly described as modification due to part-absorption of the incident radiation by atoms.

B. B. RAY.

University College of Science and Technology,

92 Upper Circular Road, Calcutta, July 10.

\section{Optical Investigations on the Formation of the Latent Photographic Image.}

THE effect of colouring of the alkali halides, such as rock salt, with $\mathrm{X}$-rays, radium radiation, or far ultra-violet radiation, is due in the light of modern knowledge to the transference of the valency electron from the chlorine to the sodium. The latter is suspected to be deposited in the lattice in the state of neutral atoms. The new absorption band, causing, for example, the yellow colour of rock salt, can be made to disappear by exposing the coloured salt to the rays it absorbs, that is, the blue-violet ones, or by heating it. A plausible theory is that in this case the backward transference of a valency electron takes place. In both cases the liberated electrons must cause the photo-conductivity effect, and this was indeed observed for the decolorising effect and studied by Pohl and Gudden and their collaborators.

On the other hand, the photo-conductivity effect was found in silver bromide and was investigated by Dr. Toy and his collaborators and also by Kirilov (Zs. f. wiss. Photographie, 1928); as Dr. Toy shows, it is closely correlated with the primary photographic process, and both are due to the same primary separation of the electron from the bromide ion.

This analogy between the processes taking place during the illumination of rock salt and silver bromide suggested some optical investigations on the absorption spectra of unexposed and exposed silver bromide, in the hope that they may contribute to the theory of formation of the latent photographical image. The silver bromide was used in the form of layers of fused salt or in large crystals, obtained by the Kyropoulos method. The following results were obtained :

(1) The silver bromide layers and crystals, when not illuminated, are lemon coloured; their absorption band lies in the blue-violet and the near ultraviolet region of the spectrum, and can be regarded as analogous to the far ultra-violet absorption band of sodium chloride (near $1800 \mathrm{~A}$.).

(2) Illumination with rays it absorbs or with $\mathrm{X}$-rays makes the layer quickly change its colour from yellow to emerald-green. The absorption band of this phase lies in the red and infra-red parts of the spectrum; it was measured by us as far as $2000 \mathrm{m \mu}$ and found to consist of a maximum at $610 \mathrm{m \mu}$ and a continuous absorption in the infra-red. It seems to be analogous to the absorption band of the yellow rock salt and can be regarded as the "absorption spectrum of the latent image " (A. Smakula, Zs. f. Phys., 59, p. 604 ; 1930).

(3) This view is supported by the observed disappearance of this 'latent image' on heating and by the action of red and infra-red radiations, which is analogous to the decolorising of the yellow salt (Herschel's phenomenon).

(4) Przibram and his collaborators have found that radiations of a given intensity cannot cause colouring of rock salt exceeding a certain maximum limit. The same is observed for the silver bromide layers. The increase of exposure does not completely compensate the decrease in the intensity (analogous to the Schwarzschild law)

(5) My investigations on the coloured rock salt, which are to appear shortly in the Zeitschrift für Physik, have led me to conclude that under some conditions (simultaneous illumination and heating) the neutral sodium atoms can aggregate into larger colloidal clumps; the absorption spectrum of such a colloidal system can be evaluated on the basis of the known theories of Maxwell-Garnett and G. Mie. The application of this evaluation to the system sodium-sodium chloride gave results which are in good agreement with the observed red, violet, and blue colour of the rock salt samples.

On the other hand, silver bromide layers, when exposed to the simultaneous action of the active and decolorising radiations, become brown or, when slightly heated, reddish brown (visual blackening); different samples may have a slightly different colour. The analogy with the change of colour of the blue rock salt by heating and other considerations leads me to assume that the brown colour is due to colloidallydistributed silver particles; the application of Mie's theory to the system silver-silver bromide seems to support this view.

M. Savostianova.

Physico-mathematical Institute,

Academy of Science,

Leningrad, July 29. 